\title{
Capacidade funcional em idosos na comunidade e propostas de intervenções pela equipe de saúde ${ }^{1}$
}

\section{Functional capacity in elderly in the community and interventions proposed in the team health}

\section{Capacidad funcional en ancianos en la comunidad y intervenciones propuestas en equipo de salud}

Adélia Yaeko Kyosen Nakatani', Luciana Barbosa da Silva", Maria Márcia Bachion"I", Daniella Pires Nunes`V

${ }^{1}$ Este trabalho contou com o apoio do Conselho Nacional de Desenvolvimento Científico e Tecnológico (CNPq).

' Doutora em Enfermagem. Professoras da Faculdade de Enfermagem da Universidade Federal de Goiás (FEN/UFG). Email: adélia@fen.ufg.br.

"Enfermeira. Ex-bolsista do PIBIC/CNPq/UFG.

II' Doutora em Enfermagem. Professoras da FEN/UFG. Email: mbachion@fen.ufg.br.

IV Acadêmica do 5o de enfermagem da UFG. Bolsista do PIBIC/CNPq/UFG.

\section{RESUMO}

Este estudo objetivou avaliar as Atividades de Vida Diária (AVD) e Atividades Instrumentais de Vida Diária (AIVD) de idosos cadastrados numa equipe da Estratégia de Saúde da Família de Goiânia (GO) e propor ações pertinentes para casos de dependência parcial ou total. Trata-se de uma pesquisa transversal e descritiva, realizada com 105 idosos em uma equipe da Estratégia de Saúde da Família. Aplicaram-se questionário e escalas de avaliação. Mediante análise de freqüência simples e percentual dos dados. Identificou-se que a maioria dos idosos era do sexo feminino, com 60 a 69 anos, casada, católica, com moradia própria, renda de até um salário mínimo, não-alfabetizada, apresentando uma a três patologias relatadas; $59,1 \%$ dos idosos apresentaram independência para AVD e 58,1\% comprometimento de AIVD. Ações pertinentes referem-se à estimulação de exercícios para ganho de força, alfabetização e motivação para a busca de autonomia. Concluise que, a maioria dos idosos consegue cuidar de si no domicílio, mas não consegue viver de forma independente com relação às AIVD.

Descritores: Idoso; Atividades cotidianas; Avaliação geriátrica; Enfermagem; Saúde da família.

\section{ABSTRACT}

This study aimed to evaluate the activities of daily life (ADL) and instrumental activities daily life (IADL) of the aged registered in a Family Health Strategy in Goiania (GO) and to propose pertinent actions in case of total or partial dependency. It is a transverse and descriptive research, accomplished with 105 elderly in a team of the Strategy of Family Health. It was applied questionnaires and evaluation scales. By analysis of simple and percentile frequency of data. It was identified that the majority was female with 60 to 69 years old, married, catholic, house-owners, having an income of up to one minimum salary, illiterate and it has been related one to three pathologies. It was verified that $59,1 \%$ of old people with maximum independence for the ADL. $58,1 \%$ presented problems in performing IADL. Pertinent actions refer to stimulate physical activity, literacy and autonomy. This study showed that the most of elderly can take care of themselves at home but they can't live independently in relation to IADL.

Descriptors: Aged; Activities of daily living; Geriatric assessment; Nursing; Health family.

\section{RESUMEN}

Este estudio ha tenido el objetivo evaluar las actividades de vida diaria (AVD) y las actividades instrumentales la vida diaria (AIVD) del anciano registrado en un Estrategia de Salud Familiar en Goiânia (GO) y para proponer las acciones pertinentes en caso de dependencia parcial o total. Fue utilizado cuestionario y escalas de evaluación. Es una investigación transversa y descriptiva, cumplido con 105 ancianos en un equipo de la Estrategia de Salud Familiar. Fueron aplicados cuestionarios y escalas de evaluación. Fue identificado que la mayoría era hembra, con 60 a 69 años, casada, católica, con casa propia, remuneración de hasta un sueldo mínimo y no alfabetizados. Se verificó $59,1 \%$ de ancianos con independencia máxima para el AVD. Un total de $58,1 \%$ de ellos estaban comprometidos con el AIVD. Las acciones pertinentes consisten en la estimulación de la actividad física, alfabetización y el empoderamiento. Los resultados evidencian que los ancianos eran capaces de cuidarse en el domicilio mas non consiguen vivir de forma independiente en relación a el AIVD.

Descriptors: Anciano; Actividades cotidianas; Evaluación geriátrica, Enfermería, salud de la familia. 


\section{NTRODUÇÃO}

Segundo o levantamento da população brasileira, realizada pelo Instituto Brasileiro de Geografia e Estatística, em 2007 nosso país tinha 17.110 .819 idosos, representando $9,3 \%$ da população total. No Estado de Goiás essa população representava 307.568 (9\%). Observa-se uma tendência no crescimento progressivo da população de idosos, na cidade de Goiânia, provavelmente devido ao processo de migração das populações da área rural para a capital ${ }^{(1)}$.

Estudo populacional, realizado em Santa Catarina, demonstra que o aumento da sobrevida acarreta maior prevalência de doenças crônicas, perdas da independência funcional e autonomia ${ }^{(2)}$. Compreende-se autonomia como a capacidade de decisão, de realizar algo com meios próprios, de forma independente. Na terceira idade, ter uma vida mais saudável significa manter ou restaurar a autonomia e a independência. Desta forma, pode-se avaliar a saúde de um idoso através dos graus de autonomia e independência com que desempenha as funções do dia-a-dia ${ }^{(3)}$.

Para mensurar a capacidade do idoso em executar atividades que o permitam cuidar de si e viver independentemente, pode ser utilizado a Avaliação Funcional que é um conjunto de dados clínicos, testes e escalas. Nesse contexto são considerados os seguintes parâmetros: equilíbrio e mobilidade, função cognitiva, condições emocionais, disponibilidade e adequação do suporte familiar e social, condições ambientais e capacidade para executar as atividades da vida diária (AVD) e atividades instrumentais de vida diária (AIVD) ${ }^{(4)}$

Capacidade funcional para atividades de vida diária consiste na aptidão que uma pessoa precisa para realizar tarefas necessárias para cuidar de si, tais como: comer, tomar banho, vestir-se, ir ao banheiro, andar, passar da cama para a cadeira, mover-se na cama, bem como apresentar controle dos esfíncteres urinário e anal ${ }^{(5)}$.

Capacidade funcional para atividades instrumentais de vida diária é a habilidade do idoso para administrar o ambiente em que vive, e inclui as seguintes ações: preparar a comida, lavar as roupas, cuidar da casa, fazer compras, ir ao médico e comparecer aos compromissos sociais e religiosos ${ }^{(5)}$.

Existem inúmeras escalas que servem para quantificação da capacidade para executar as AVD e AIVD. Devem ser breves, simples e de fácil aplicação, para que atinjam as metas, quais sejam: servir como instrumento rápido de avaliação, triagem e estratificação de risco, e possam ser utilizadas por pessoal de variada formação profissional e em qualquer unidade básica de saúde.

A avaliação funcional dos idosos é importante e deve ser incorporada a qualquer roteiro de entrevista feita pelos profissionais de saúde, pois possibilita identificar tanto as limitações como as potencialidades desse grupo populacional, para então traçar o plano de ação, com as devidas medidas preventivas, terapêuticas e reabilitadoras.

As ações relacionadas à saúde do idoso devem ter como objetivo mantê-lo na comunidade de forma autônoma e independente, vinculado ao sistema de saúde local.

Para cumprir os propósitos de atender as reais necessidades locais, a Estratégia de Saúde da Família (ESF) deve realizar estudo diagnóstico dos grupos populacionais de sua área de abrangência. Assim, poderá planejar ações globais, e não apenas atender à demanda espontânea. Poderá ainda avaliar os resultados de suas ações, comparando as condições de saúde ao longo do tempo.

Este estudo teve como objetivos caracterizar o perfil sócio-demográfico e de saúde, avaliar as atividades de vida diária (AVD) e atividades instrumentais de vida diária (AIVD) de pessoas idosas cadastradas em área de abrangência de equipe da Estratégia de Saúde da Família de Goiânia, GO, e propor, no contexto da Estratégia de Saúde da Família, medidas de intervenção frente ao diagnóstico da capacidade em realizar AVD e AIVD identificada entre idosos.

\section{METODOLOGI A}

Trata-se de um estudo transversal e descritivo, realizado com idosos da área de abrangência de uma equipe da Estratégia de Saúde da Família (ESF) do Município de Goiânia/GO. Foi selecionada, aleatoriamente, a equipe 5, localizada no Centro de Atendimento Integrado de Saúde (CAIS) Amendoeiras, no Distrito Sanitário Leste, onde a Faculdade de Enfermagem da Universidade Federal de Goiás desenvolve atividades de ensino, pesquisa e extensão.

A população foi composta de 107 idosos, com idade igual ou superior a 60 anos, que corresponde ao total de pessoas, nessa faixa etária, cadastradas pela equipe 5, do ESF do Parque das Amendoeiras. Após serem esclarecidos sobre a pesquisa, 105 idosos concordaram em participar do estudo e assinaram o Termo de Consentimento Livre e Esclarecido, de acordo com a Resolução 196/96 do Conselho Nacional de Saúde, relativa à pesquisa envolvendo seres humanos ${ }^{(6)}$.

A coleta de dados foi realizada no domicílio de cada idoso, no período de novembro de 2001 a maio de 2002, mediante aplicação de questionário sobre o perfil social, econômico, demográfico e de saúde dos idosos, com itens relativos à idade, gênero, estado civil, religião, escolaridade, renda, condições de moradia e doenças auto-referidas. 
Foram utilizadas, ainda, duas escalas de avaliação funcional: uma para mensurar a capacidade em executar as atividades de vida diária ${ }^{(7)}$ e outra para medir a capacidade em executar as atividades instrumentais de vida diária ${ }^{(8)}$. Trata-se de escalas desenvolvidas na década de 1960, de uso consagrado entre estudiosos de avaliação da capacidade funcional, não só na área de Gerontologia e Geriatria $^{(2,4)}$, como também de outras áreas ${ }^{(9-10)}$.

A escala de avaliação das atividades de vida diária $^{(7)}$ inclui o exame da capacidade do idoso para alimentar-se, higienizar-se, vestir-se e arrumar-se, deambular, transferir-se da cama para a cadeira, mover-se no leito, subir e descer escadas e ter continência nas micções e evacuações. A pontuação obtida pode variar entre máxima dependência $(0$ pontos) e máxima independência (100 pontos). Idosos com pontuação abaixo de 70 necessitam de supervisão ou assistência, para a maioria das atividades.

A escala de avaliação das atividades instrumentais de vida diária ${ }^{(8)}$ engloba a análise da capacidade do idoso para: preparar suas refeições, realizar tarefas domésticas, lavar a roupa, manusear medicamentos, usar o telefone, manusear dinheiro, fazer compras, usar meios de transporte. A mesma forma, pontuação obtida leva ao julgamento de independência (24 pontos), dependência total (8 pontos) ou dependência parcial ( 9 a 23 pontos).

Os dados foram organizados e tabulados, utilizando-se um software de estatística (Epi Info versão 6.2) e as variáveis analisadas de forma descritiva, mediante freqüências simples e percentual, média, moda, mediana e quartis.

O estudo foi aprovado pelo Comitê de Ética em Pesquisa (COEP), da Universidade Federal de Goiás, protocolo no 26/2001.

\section{RESULTADOS E DISCUSSÃO}

Foram abordados todos os 107 idosos cadastrados na equipe 5, do ESF do Parque das Amendoeiras. Dois idosos se recusaram a participar da pesquisa. Assim, foram entrevistados 105 idosos, o que equivale a $98,13 \%$ do total. A faixa etária dos sujeitos variou de 60 a 94 anos, sendo a média de 69,7 anos (Tabela 1). Esses dados corroboram os achados em Goiás, onde a expectativa de vida, atualmente, é de, aproximadamente, $69 \operatorname{anos}^{(11)}$.

Tabela 1: Características sócio-demográficas dos idosos cadastrados em uma equipe de Saúde da Família. Parque das Amendoeiras. Goiânia, 2001-2002 (n=105).

\begin{tabular}{|l|c|c|}
\hline Características & $\mathbf{n}$ & $\%$ \\
\hline Faixa etária & & \\
\hline $60-69$ anos & 61 & 58,0 \\
\hline $70-79$ anos & 32 & 30,5 \\
\hline$\geq 80$ anos & 12 & 11,5 \\
\hline Sexo & & \\
\hline Feminino & 57 & 54,3 \\
\hline Masculino & 48 & 45,7 \\
\hline Estado Civil & & \\
\hline Casado/ união consensual & 53 & 50,5 \\
\hline Viúvo & 36 & 34,3 \\
\hline Solteiro & 16 & 15,2 \\
\hline Religião & & \\
\hline Católica & 64 & 60,9 \\
\hline Evangélica & 32 & 30,5 \\
\hline Espírita & 05 & 4,8 \\
\hline Ausente & 04 & 3,8 \\
\hline Instrução & & \\
\hline Não-alfabetizado & 56 & \\
\hline Alfabetizado & 49 & \\
\hline Renda & & \\
\hline I salário & 90 & \\
\hline 1 a 3 salários & 10 & 86,7 \\
\hline$>3$ salários & 5 & \\
\hline Moradia & & \\
\hline Própria & 99 & \\
\hline Alugada & 6 & \\
\hline
\end{tabular}


Houve predominância do sexo feminino de forma semelhante a encontrada no Município de Aparecida de Goiânia/GO(12), contudo com taxa menor do que a identificada em outros estudos fora do Estado de Goiás ${ }^{(13-15)}$.

A maioria dos idosos vivia em companhia do cônjuge/companheiro (50,5\%). Esse resultado, também, foi verificado em outra pesquisa ${ }^{(16)}$, abrangendo a população idosa de quatorze municípios do Estado de Goiás. Os idosos viúvos representaram $34,3 \%$ da população entrevistada, sendo que $16,7 \%$ viviam sozinhos e $83,3 \%$ com familiares, residindo, em média, com 3,06 pessoas. Os idosos solteiros somaram $15,2 \%$, sendo que $31,2 \%$ viviam sozinhos e $68,8 \%$, morava, em média, com 2 pessoas da família.

Os dados revelaram que a maioria dos idosos professava algum tipo de religião, sendo $60,9 \%$ católicos. Esta característica também foi observada em outro estudo, que evidenciou a experiência, a maturidade, os problemas vividos e a maior disponibilidade de tempo como fatores que conduzem os idosos a uma maior aproximação com a espiritualidade ${ }^{(12)}$.

Verificou-se que $53,3 \%$ dos entrevistados eram analfabetos, e os alfabetizados somaram $46,7 \%$. Entre eles, $48(97,9 \%)$ estudaram de 1 a 5 anos, sendo a média igual a 3,04 anos e a moda igual a 4 anos. Apenas uma idosa referiu ter terminado o ensino médio.

Uma das características marcantes da população estudada foi a pobreza $(85,7 \%$ tinha renda de até um salário mínimo por mês). A renda destes idosos é menor em comparação a média nacional ${ }^{(11)}$.

O Parque das Amendoeiras encontra-se na periferia de Goiânia, e grande parte dos idosos entrevistados relatou que este bairro consistia em área invadida pelos moradores, até a Prefeitura distribuir as respectivas escrituras, explicando, assim, porque a maioria possui moradia própria $(94,3 \%)$, mesmo com uma renda mensal tão baixa.

Mediante afirmação de diagnóstico médico pregresso $65,7 \%$ alegaram sofrer de uma a três morbidades, $26,7 \%$ não apresentavam nenhuma doença e $7,6 \%$, afirmaram conviver com mais de três enfermidades. Comparando-se estes achados com a literatura, verificou-se que os participantes apresentam índices de morbidade inferiores a idosos de outras regiões do Brasil. Em estudo realizado na região sul, $40,3 \%$ relataram ter três ou mais enfermidades crônicas $^{(2)}$. Na região sudeste $5,5 \%$ dos idosos referiram não sofrer de nenhuma doença e $74,9 \%$ de uma a cinco ${ }^{(13)}$.

Entre as ocorrências de maior freqüência, a hipertensão arterial foi referida por $46(43,8 \%)$ idosos, diabetes por $16(15,2 \%)$ e $13(12,3 \%)$ informaram osteoporose. Trata-se de doenças crônicas de maior prevalência em idosos, como mostra estudos ${ }^{(3,17)}$, que identificaram o perfil de morbidade de idosos em algumas cidades brasileiras, encontrando prevalências elevadas para hipertensão arterial, doenças do coração, reumatismo, doenças mentais, diabetes, doença cérebro vascular e doenças respiratórias.

Como pode ser visto na Tabela 2 , entre os idosos avaliados, $62(59,1 \%)$ eram independentes para realizar todas as AVD, obtendo pontuação igual a 100 na escala de avaliação(7). Contudo, 43 $(40,9 \%)$, possuíam algum grau de dependência para realizar uma ou mais atividades. A pontuação média alcançada por esses idosos, na escala de Atividades de Vida Diária ${ }^{(7)}$, foi de 90 pontos, sendo a moda igual a 95. O número de atividades comprometidas variou de 1 a 8 , sendo a média aritmética igual a 1,6 ; o primeiro quartil igual a 1 , o segundo quartil igual a 1 e o terceiro quartil igual a 3 atividades. A moda encontrada foi de 1 atividade.

Tabela 2: Capacidade em realizar as atividades de vida diária (AVD) entre os idosos cadastrados em uma equipe de Saúde da Família. Parque das Amendoeiras. Goiânia, 2001-2002 ( $n=105)$.

\begin{tabular}{|c|c|c|}
\hline Capacidade para realizar as AVD & $\mathbf{n}$ & $\mathbf{\%}$ \\
\hline Independente & 62 & 59,1 \\
\hline Dependente para 1 a 3 AVD & 40 & 38,1 \\
\hline Dependente para 4 a 6 AVD & 2 & 1,9 \\
\hline Dependente para 7 a 9 AVD & 1 & 0.9 \\
\hline Dependente para todas AVD, ou seja, 10 & 0 & 0 \\
\hline TOTAL & $\mathbf{1 0 5}$ & $\mathbf{1 0 0}$ \\
\hline
\end{tabular}

Estudo realizado no oeste de Minas Gerais identificou que a possibilidade de dependência para AVD é de 3,1 vezes na faixa etária de 80 anos e 1,5 vezes na de 70-90 anos, quando comparada à faixa etária de 60 a 69 anos $^{(14)}$. Outro estudo, em São Paulo demonstrou que a chance de ter dependência para AVD na faixa etária de 65 a 69 anos é de 1,9 vezes, aumentando até 36 vezes entre os idosos de mais de 80 anos e que o nível baixo de escolarização apresentaram cerca de cinco vezes mais chance de ter dependência moderada/grave ${ }^{(15)}$.

Em uma outra pesquisa, que abordou a população, no município de São Carlos, identificou que mais da metade dos idosos precisa de ajuda para 
a realização de pelo menos 1 atividade do autocuidado $(\text { AVD })^{(13)}$. Comparando os achados, podemos dizer que os participantes da presente pesquisa estão em melhores condições.

Entre os participantes, no geral, 28,6\% referiram dificuldade para o controle do esfíncter urinário. Esse contingente representa 69,8\% entre os idosos com algum comprometimento nas AVD.

Do total de idosos, na amostra, 6,6\% alegaram dificuldade de controle do esfíncter anal, que representa $16,3 \%$ entre aqueles com algum comprometimento nas AVD.

Os idosos que apresentam incontinência, em geral, passam constrangimentos, levando-os à restringirem-se ao lar. Esta condição contribui para agravos como solidão, depressão e alteração cognitiva. Cabe à equipe de profissionais avaliar o motivo da incontinência e traçar condutas com vistas a recuperar o controle dos esfíncteres.

Com relação à incontinência urinária, alguns procedimentos para o controle das micções são: ensinar o idoso a inibir voluntariamente as contrações da bexiga ou contrair os músculos do assoalho pélvico de forma a manter a continência; aconselhar o uso de coletores externos (sistema de coletor externo e fraldas geriátricas) ${ }^{(18)}$.

Com relação à incontinência nas evacuações a equipe de saúde pode orientá-lo quanto à realização de exercícios de contração da musculatura anal com objetivo de hipertrofiar o esfíncter externo do ânus e a musculatura do assoalho pélvico ${ }^{(17)}$, resgatando o controle das eliminações, e facilitando o dia-a-dia do idoso e da família, poupando-o sempre, que possível, de constrangimentos.

Entre os participantes, outra área comprometida foi a capacidade para subir e descer escadas, relatada por $12,4 \%$ do total de idosos, na amostra, que representa $30,2 \%$ entre aqueles com algum comprometimento nas AVD. Este achado indica perdas motoras maiores do que as encontradas em outro estudo(16), em que subir escadas representou 4,6\% das limitações, na execução das AVD.

A dificuldade para subir e descer escadas, dentro ou fora do domicílio, prejudica a sua autonomia para ir e vir. O idoso precisará de ajuda para ir a serviços de saúde, supermercados, lojas, instituições religiosas, casas de parentes e amigos, e a família nem sempre estará disponível para acompanhá-lo. Caso isto ocorra sistematicamente, poderá comprometer a socialização do idoso.

As características de estrutura física para evitar agravos a idosos independentes ou dependentes incluem escadas com corrimão de cor contrastante com a parede, piso antiderrapante com faixa de sinalização de fácil visualização, para facilitar a orientação e a locomoção ${ }^{(18)}$. Cada família, dentro de suas possibilidades, precisa adequar a área do domicílio, para garantir o deslocamento seguro do idoso. Por outro lado, as políticas públicas devem intensificar ações para assegurar condições de acesso e de trânsito para os idosos, garantindo-Ihes o direito de ir e vir.

Além do mais, podem ser realizadas medidas de terapia física, orientadas e prescritas por profissionais, visando a conservação e o aumento da força muscular, treinamento do andar, aumento da mobilidade articular, correção de deformidades articulares e melhora do equilíbrio(18).

Isto se torna relevante à medida que diversos estudos têm projetado um grande crescimento da população idosa funcionalmente incapacitada, sendo que o número dessas pessoas dependentes nas AVD dobrará na segunda ou terceira década, do próximo século(3)

Diante dessa realidade é preciso que a equipe de saúde local se preocupe em preparar os cuidadores, formais e informais, com conhecimentos sobre o processo de envelhecimento e cuidados básicos, que devem ser realizados frente a cada necessidade, para que os idosos se beneficiem através do atendimento domiciliar com qualidade.

Com relação às AIVD pudemos perceber uma inversão dos achados, em comparação com as AVD, ou seja, 61 idosos $(58,1 \%)$ possuíam algum grau de dependência, para administrar o seu ambiente e lidar com a vida social, sendo $57,1 \%$ com dependência parcial e $1 \%$ com dependência total.

Os idosos parcialmente dependentes para as AIVD apresentaram pontuação média na escala de avaliação de Atividades Instrumentais de Vida

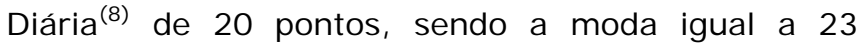
pontos. Um único idoso totalmente dependente para as AIVD apresentou pontuação igual a 8.

A perda da independência funcional, quase sempre se traduz em perda de autonomia. À medida que um idoso demonstra algum grau de dependência para administrar seus recursos financeiros ou adquirir alimentos e bens de consumo básicos, começa a ter sua autonomia prejudicada. Assim, outras pessoas passarão a gerir seus bens financeiros, fazer suas compras, decidir sobre o que o idoso deve ou não deve fazer ${ }^{(19)}$

Entre os itens das AIVD nos quais os idosos apresentaram algum grau de dependência, conforme pode ser visto na Tabela 3, identificamos: lavar roupa $(38,1 \%)$, trabalho doméstico - limpar casa e tirar poeira $(32,4 \%)$, utilizar o telefone $(30,5 \%)$, usar os meios de transporte $(24,5 \%)$, fazer compras $(17,1 \%)$, manuseio de dinheiro $(16,1 \%)$, tomar medicação $(12,4 \%)$ e preparar as refeições $(7,6 \%)$. Entre os 61 idosos dependentes para as AIVD, alguns referiram dependência em mais de uma atividade. 
Tabela 3: Distribuição do nível de dependência para realizar as atividades instrumentais de vida diária em idosos de uma equipe de Saúde da Família. Parque das Amendoeiras. Goiânia, 2001-2002 (n=61).

\begin{tabular}{|l|c|}
\hline \multicolumn{1}{|c|}{ Dependência em realizar as AIVD } & n \\
\hline Preparar as refeições & 6 \\
\hline incapaz de preparar as refeições & 2 \\
\hline necessita de ajuda para preparar as refeições & \\
\hline Trabalho doméstico & 27 \\
\hline Capaz de realizar apenas trabalho doméstico leve & 7 \\
\hline incapaz de realizar qualquer trabalho doméstico & \\
\hline Lavar roupa & 35 \\
\hline Capaz de lavar peças pequenas & 5 \\
\hline incapaz de lavar qualquer peça & \\
\hline Tomar medicação & 11 \\
\hline necessita de lembretes para tomar a medicação & 2 \\
\hline incapaz de tomar medicação & \\
\hline Utilizar o telefone & 26 \\
\hline necessita de ajuda ou aparelho especial para discar & 6 \\
\hline incapaz de usar o telefone & \\
\hline Manuseio de dinheiro & 12 \\
\hline necessita de ajuda com cheques e pagamentos de contas & 5 \\
\hline incapaz de lidar com dinheiro & \\
\hline Compras & 14 \\
\hline necessita de supervisão para fazer compras & 4 \\
\hline incapaz de realizar as compras, mesmo com supervisão & \\
\hline Uso de meios de transporte & 20 \\
\hline necessita de ajuda quando viaja de ônibus, trens, metrôs e táxi & 6 \\
\hline incapaz de utilizar qualquer meio de transporte & \\
\hline
\end{tabular}

A incapacidade na realização de atividades como lavar roupa, limpar a casa pode comprometer a manutenção da higiene do domicílio e do próprio idoso. Pode levar a sentimentos de culpa ou baixa auto-estima dada a incapacidade de não mais conseguir realizar tais atividades, de forma independente.

No momento das entrevistas muitos idosos do sexo masculino disseram que não lavam roupas e nem fazem serviços domésticos por comodidade, pois tais atividades sempre foram realizadas inicialmente pelas mães e posteriormente pelas esposas. Entretanto, quando questionados se seriam capazes de realizar as tarefas, na ausência de uma mulher, responderam que sim.

Algumas incapacidades evidenciadas na amostra como: tomar medicação, discar o telefone, manusear dinheiro, fazer compras e usar meios de transportes, associam-se ao analfabetismo conforme os relatos dos idosos entrevistados. Somam-se a isso as dificuldades de acompanharem as transformações sociais, econômicas, tecnológicas devido ao afastamento progressivo da vida social.

Atualmente, as pesquisas voltadas ao envelhecimento, têm dado ênfase à necessidade da implementação de serviços de promoção à saúde, preservação da vida e recuperação de doenças, de modo que a capacitação das pessoas para cuidar de idosos se reflita no desempenho do autocuidado, na manutenção da autonomia e independência dos idosos $^{(5,20)}$.

Nesse sentido, cabe às equipes da ESF implementar ações, visando potencializar as funções cognitivas remanescentes de alterações decorrentes do próprio envelhecimento ou de processos patológicos. Cabem ainda, ações de promoção de convívio social, adaptação da sociedade às deficiências do idoso, maior envolvimento da família na sua estimulação para manutenção de uma vida produtiva, por mais tempo.

As limitações, dependência/ independência, adaptações na realização de muitas atividades pelos idosos podem decorrer do processo de envelhecimento ou da evolução de doenças crônicodegenerativas.

É necessário que ocorra uma reestruturação da atuação da equipe de saúde local no sentido de prevenção, diagnóstico precoce e tratamento das doenças crônicas e das incapacidades associadas no adulto e no idoso, em particular. A concepção do trabalho da equipe deve ser a de procurar manter a autonomia do indivíduo, uma vez que sua perda acarreta alterações na integridade da pessoa, e aumenta sobrecarga da família e do sistema de saúde ${ }^{(20)}$. 


\section{CONCLUSÕES}

Quanto ao perfil social, econômico, demográfico e de saúde dos idosos concluímos tratar-se de uma população predominantemente feminina, católica, com idade de 60 a 69 anos, não alfabetizada, com renda mensal menor que um salário mínimo, residente em moradia própria (proveniente de área invadida).

Observamos que $59,1 \%$ dos idosos eram independentes para as AVD. Entretanto, em relação à execução as AIVD 58,1\% eram dependentes em uma ou mais atividades. Isto significa que a maioria dos idosos consegue cuidar de si no domicílio, mas não consegue viver de forma independente no seu meio ambiente externo.

Diante dessa realidade, a equipe de saúde deve desenvolver ações em direção à promoção da saúde das pessoas no processo de envelhecimento, com objetivo de despertar as autoridades envolvidas no Sistema de Saúde para desenvolverem políticas públicas que viabilizem autonomia às populações idosas.

Além disso, deve implementar ações de promoção da saúde para os idosos que ainda se mantêm independentes. Por outro lado, são necessárias ações de prevenção de incapacidade física, mental e social e reabilitação para aqueles que possuem algum grau de dependência.

A velhice é um "estado de ser" como outro qualquer, deve ser vivida com intensidade, autonomia e prazer. Cabe ao próprio indivíduo, a sua família, a sua comunidade e ao Estado desenvolverem ações para que esta importante etapa da vida seja uma fase em que as pessoas continuem sendo úteis e produtivas.

\section{REFERÊNCI AS}

1. Ministério do Planejamento, Orçamento e Gestão; Instituto Brasileiro de Geografia e Estatística. Contagem da População 2007. Rio de Janeiro (Brasil): Ministério do Planejamento, Orçamento e Gestão; 2007.

2. Santos KA, Koszuoski R, Dias-da-Costa, JS, Pattussi MP. Fatores associados com a incapacidade funcional em idosos do município de Guatambu, Santa Catarina, Brasil. Cad. Saúde Pública. 2007; 23(11):2781-88.

3. Paschoal, SMP. Autonomia e independência. In: Papaléo Netto M. Gerontologia: a velhice e o envelhecimento em visão globalizada. São Paulo: Atheneu; 2002. p.313-23.

4. Costa EFA, Porto CC, Almeida JC, Cipullo JP, Martin JFV. Semiologia do idoso. In: Porto CC. Semiologia Médica. Rio de Janeiro: Guanabara Koogan; 2005. p.154-83.

5. Ministério da Saúde. Envelhecimento e saúde da pessoa idosa. Brasília (Brasil): Ministério da Saúde; 2006. n.19.

6. Ministério da Saúde; Conselho Nacional de Saúde. Resolução № 196/96 - Normas regulamentadoras de pesquisa envolvendo seres humanos. Brasília (Brasil): Ministério da Saúde; 1996.

7. Mahoney FI; Barthel D. Functional evaluation: the Barthel index. Maryland State Med Journal. 1965; 14:56-61.

8. Lawton MP, Moss M, Fulcomer M, Kleban MH. A Research and service-oriented multilevel assessment instrument. J Gerontol 1982;37(1):91-9.

9. Rocco FM, Luz FHG, Rossato AJ, Fernandes AC, Oliveira ASB, Beteta JT, et al. Motor function evaluation in merosin-deficient congenital muscular dystrophy children. Arq. Neuro-Psiquiatr. 2005; 63(2): 298-306.

10. Rodrigues JE, Sá MS, Alouche SR. Perfil dos pacientes acometidos por AVE tratados na clínica escola de fisioterapia da UMESP. Revista Neurociências [Internet]. 2004 [cited 2009 feb 12];12(3):117-122. Available from: http://www.unifesp.br/dneuro/neurociencias/vol12_3 Lave tratados. htm

11. Ministério do Planejamento, Orçamento e Gestão; Instituto Brasileiro de Geografia e Estatística. Pesquisa Nacional por Amostra de Domicílios. Rio de Janeiro (Brasil): Ministério do Planejamento, Orçamento e Gestão; 2005.

12. Araujo MAS, Nakatani AYK, Silva LB, Bachion MM. Perfil do idoso atendido por um programa de saúde da família em Aparecida de Goiânia - Go. Revista da UFG [Internet]. 2003 [cited 2009 feb 12]; 5(2). Available from: http://www.proec.ufg.br/revista_ufg/idoso/perfil.html 13. Feliciano AB, Moraes SA, Freitas ICM. O perfil do idoso de baixa renda no município de São Carlos, São Paulo, Brasil: um estudo epidemiológico. Cad. Saúde Pública. 2004; 20(6):1575-85.

14. Giacomin KC, Uchôa E, Firmo JOA, Lima-Costa MFF. Projeto Bambuí: um estudo de base populacional da prevalência e dos fatores associados à necessidade de cuidador entre idosos. Cad. Saúde Pública. 2005; 21(1):80-91.

15. Rosa TEC, Benício MHA, Latorre MRDO, Ramos LR. Fatores determinantes da capacidade funcional entre idosos. Rev. Saúde Públ. 2003;37(1):40-8.

16. Soares ERB, Rocha KB, Moraes MER. Diagnóstico da Situação da População idosa em quatorze municípios do estado de Goiás [monograph]. Goiânia: Faculdade de Enfermagem/UFG; 2002.

17. Araújo SEA, Habr-Gama A. Incontinência fecal. In: Manual de Condutas Médicas/Instituto para o Desenvolvimento da Saúde. Brasília: Ministério da Saúde; 2002. p.388-90.

18. Ministério da Previdência e Assistência Social. Idosos: Problemas e cuidados básicos. Brasília (Brasil): Ministério da Previdência e Assistência Social; 1999.

19. Ministério da Saúde. Cadernos de Atenção Básica - Programa de Saúde da Família. Instabilidade Postural e Queda. Brasília (Brasil): Ministério da Saúde; 2000.

20. Veras $R$ A. frugalidade necessária: modelos mais contemporâneos. Cad. Saúde Pública. 2004; 20(5): 1141-59.

Artigo recebido em 10.03.08.

Aprovado para publicação em 31.03.09. 